\title{
Studying and Simulation for Different Geometry of Nanoholes Array (NHA) in Surface Plasmons Biosensor
}

\author{
Mohanad Hasan Ali Aljanabi ${ }^{1,2}$ \\ ${ }^{1}$ Department of Electrical and Computer Engineering, Istanbul Kemerburgaz University, Istanbul \\ ,Turkey. \\ ${ }^{2}$ Department of Electrical Power Techniques Engineering, Technical College /Al-Mausaib,Al-Furat Al-Awsat \\ Technical University, Iraq
}

\begin{abstract}
The possibility to limit and manipulate photons at nanometer scales attracted a lot of interest for exciting applications from subwavelength in biosensors and optoelectronics devices, the sensor optical properties, however; are complex due to two resonances through propagating and localized surface plasmons. The optical properties of surface plasmons(SPs) at the resonant wavelength is depending on the geometrical nanostructure of materials. In this articles, we used different geometry of nanoholes array, 4 and 9 nanoholes array in a metallic film gold nanoparticles with different thickness $(20,50,100) \mathrm{nm}$ on SiO2 substrate with refractive index 1.46, we designed two different geometries; 4- holes: hole radius $r l=200 \mathrm{~nm}$, period $\mathrm{pl}=600$ $\mathrm{nm}$; and 9- holes : $r 2=100 \mathrm{~nm}$, period $\mathrm{p} 2=300 \mathrm{~nm}$.

Transmission and reflection spectrum have been calculated and simulated by FDTD Lumerical program. From results are observed the effect of thickness is interesting, transmission is increased at ( $t=20 \mathrm{~nm})$ for two arrays. Furthermore, the number of hole and its area has an influence on optical transmission and other parameters $(E, H, R e f)$ which are characteristics of design of metallic nanostructure.

We can see that there is a peak value of the wavelength at $519 \mathrm{~nm}$ approximately to $73 \%$ strong light transmission with 4-NHA in the other hand wavelength of $519 \mathrm{~nm}$ transmission is 45\% with 9-NHA. strong light transmission is hopeful for many applications(Biosensors devices).
\end{abstract}

Keywords: Plasmonics .Optical transmission. Biosensors . FDTD. Localized surface plasmons. Nanoholes array. Geometrical parameters.

\section{1-Introduction}

Surface Plasmons (SPs) are collective oscillations of free electrons which are excited by light or photon (from visible to IR) [1]. Firstly SPP involves on one side the coherent oscillation of the conduction electrons at the interface inside the metal, and on the other side the propagation of an electromagnetic wave along the surface, inside the dielectric as equations (1\&2) as shown in figure (1), we find the values of wave number and Surface Plasmon frequency $\omega_{s p}$ as shown in figure (2) , as [2,3]

$$
\begin{array}{r}
\beta=\frac{\omega}{c} \sqrt{\frac{\varepsilon_{m} \varepsilon_{d}}{\varepsilon_{m}+\varepsilon_{d}}} \\
\omega_{s p}=\frac{\omega_{p}}{\sqrt{1+\varepsilon_{d}}}
\end{array}
$$


Secondly, Localized surface Plasmons (LSPs) are non-propagating excitations of the conduction electrons of metallic nanostructures coupled to the electromagnetic field. LSPs are excited at certain resonance frequencies (or resonance wavelengths, typically in the visible region), resulting in scattering and absorption at these frequencies for example optical nanoantennas [4] A Plasmon is a quantized plasma oscillation, and the motion of the collective oscillation can rightly support electromagnetic (EM) waves at optical frequencies. The LorentzDrude model equation of motion is:-

$$
\vec{F}=m \vec{a}=m \frac{d^{2} x}{d t^{2}}=-q E
$$

The dipole moment per unit volume, is:-

$$
\begin{gathered}
P=-n q x=-\frac{n q^{2}}{m \omega^{2}} E \\
\omega_{p}^{2}=\frac{n q^{2}}{\varepsilon_{o} m}
\end{gathered}
$$

Nanoholes array display high transmission due to the matching between the incident light and SPRs through the holes at the surface of the metallic film [5,6]. Thin metallic films patterned with a nanoholes array also support surface plasmons excitation due grating coupling, and the resonance condition for Plasmon excitation depends on refractive index of the surrounding medium [7].

EOT (An extraordinary optical transmission) supported by a subwavelength periodic nanoholes array patterned on thin film of metal $[8,9]$.

Some geometrical coefficients like a thickness of metal and substrate; the hole diameter also the number of periodic holes and metal properties can effect on EOT spectra because the surface plasmons that will be excited by incident light is affected by changing the size of hole and it have been confirmed that any change in the size of the holes lead to a small variations in transmission efficiency and peak positions [10-14]. Je Hong provided that the transmission characteristics is a function of film thickness of metallic hole array samples which is prepared with different hole periodicity, size and shape on a silica substrate and shows highly transmission efficiency at small thickness of gold metal $[15,16]$. Plasmonic nanostructures have been extensively investigated both for scientific interest in their unique optical properties and for practical applications, such as optoelectronic devices, sensors, biomedical treatment; and so on [ 17,18].

Since plasmonic sensing requires simply an optical transmission spectrum, or only the transmitted intensity at one or a few wavelengths [19].

In this study we presented the differences between two holes arrays of 4 and 9 holes for different thickness $(20,50,100) \mathrm{nm}$ to characterize the optical properties for surface plasmons metals and to determined from these parameters the affects geometry and shape on sensors applications and optoelectronic devices .

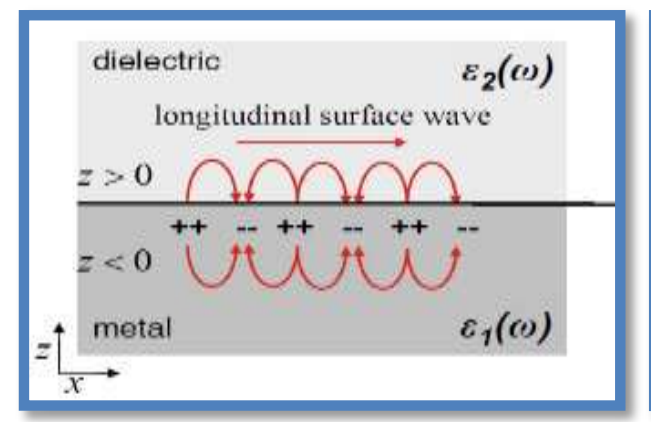

Fig.1 "Schematic of a Surface Plasma wave (SPPs) at a metal-dielectric interface"[3].

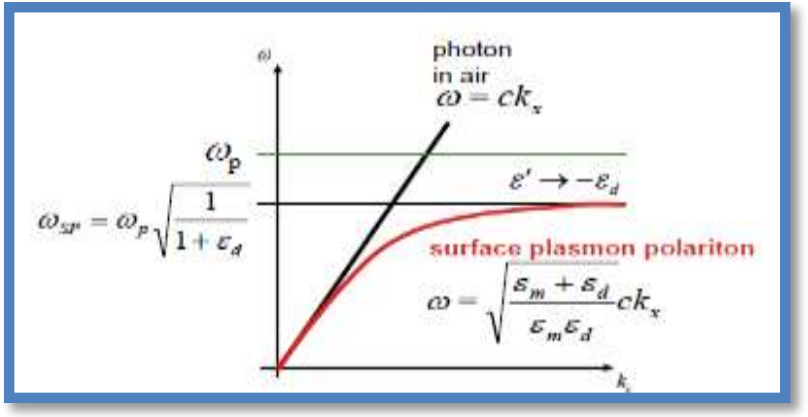

Fig 2. "How to find the frequency surface plasmons at air and dielectric" [2]. 


\section{Method of Analysis}

Two designs of nanoholes array (NHA) of 4 and 9 nanoholes which they patterned with periodic array of nanoholes with radius $r_{1}, r_{2}$ for 4 and 9 respectively in thin film of gold in different thickness for each design $(20,50,100) \mathrm{nm}$ which based on $\mathrm{SiO}_{2}$ substrate with refractive index $(\mathrm{n}=1.46)$ and thickness fixed in two cases $(\mathrm{t}=950 \mathrm{~nm})$.

These designs are performed as $2 \times 2$ array with $\mathrm{P}_{1}=600 \mathrm{~nm}$ and $\mathrm{r}_{1}=200 \mathrm{~nm}$ in the other hand $3 \times 3$ array with $\mathrm{P}_{2}=600 \mathrm{nmand} \mathrm{r}_{2}=200 \mathrm{~nm}$, with consideration that these holes are patterned on the same direction of a sample $\mathrm{Au} / \mathrm{SiO}_{2}$ as shown in figure (3a-b).

In this work we use FDTD method that deals with Maxwell's equations in surface plasmons ,this technique is helpful to analyze the interaction of incident light with structure (NHAs) and by Durde model is computed the noble metals which fits the literature data from Palik [20]. From these arrays the transmission and reflection have been calculated by such program and considered magnetic field intensity $(\mathrm{H})$ and electric field intensity (E) [21]. In addition, there was researches about different geometry and shapes like square and hexagonal lattice, grating and grid thin film of metal to develop biological sensing performance .

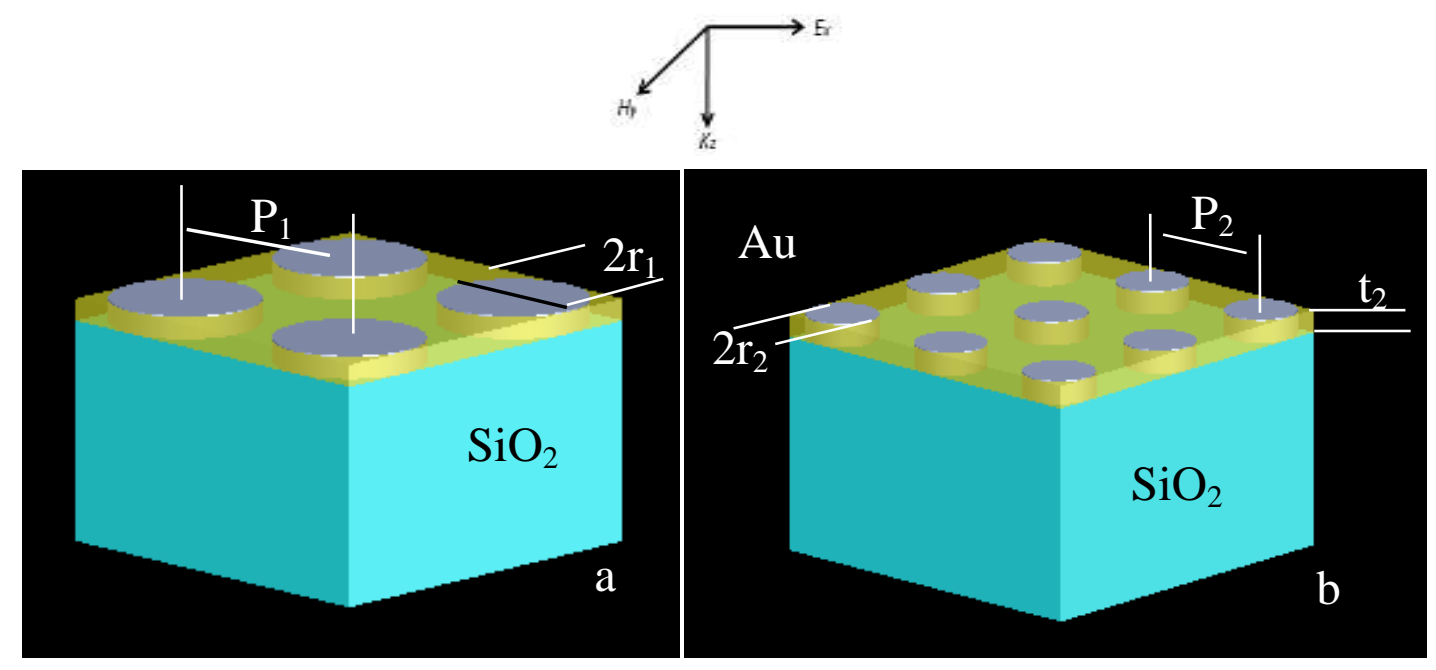

Fig.3 a-b "Schematic View of 4 and 9 NHA Respectively, Including the Polarization \& Propagation

Direction of the Incident Wave and Geometrical Parameters: T, R, P".

\section{Results and discussion}

In this work, we used FDTD by Lumerical solution Inc. to design a simulation of nanoholes array of 4 and 9-NHA in different periodic array and diameters of holes patterned on thin film of gold based on silicon dioxide, $\mathrm{SiO}_{2}$ substrate $(\mathrm{n}=1.46)$ by this program the interaction of incident light wavelength $(\lambda=350-750)$ $\mathrm{nm}$ with nanoholes array structure is analyzed. From the simulation FTDT and design of NHA, the asymmetric and anti symmetric boundary conditions were considered for the $\mathrm{x}$ - and y-directions, respectively, and we study the transmission properties at normal incident of electromagnetic wave through the sub wavelength nanoholes structure. For 4-NHA, the FDTD simulations showing that transmission efficiency reached about $73 \%$ in visible. 

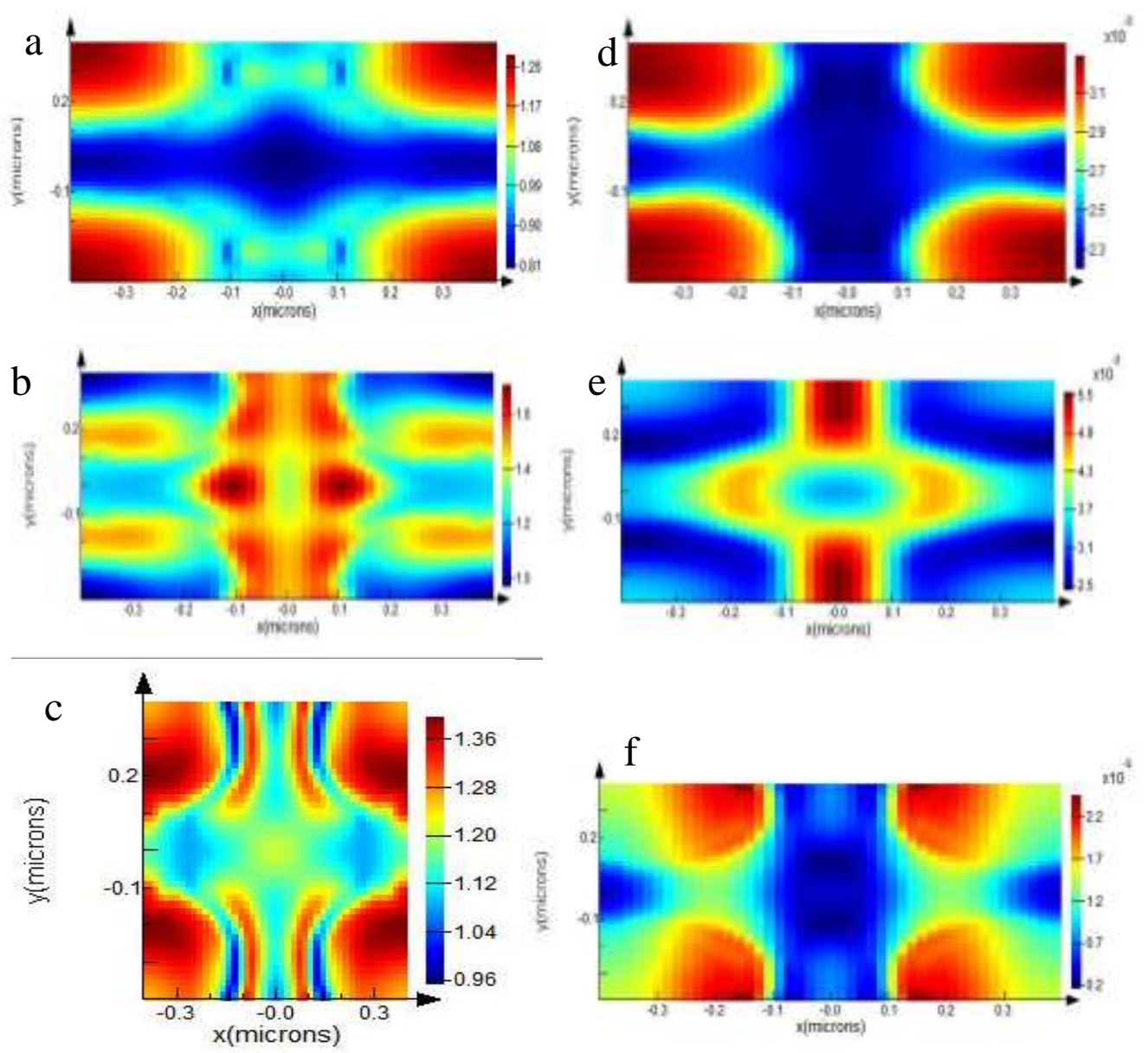

Fig (3 ) "Field Distributions for H and E of 4-NHA with Varying Thickness of Au: $(20,50,100)$ Nm"

From fig.(3) the E distributions for 4-holes array with period $\mathrm{P}_{1}=600 \mathrm{~nm}$ are shown in fig.(3a-c), we can see that $\mathrm{E}$ at $\mathrm{t}=50 \mathrm{~nm}$ is larger than the other thickness $(20,100) \mathrm{nm}$ corresponding to the two modes of transmission in fig. ( 5c) to determined the near field enhancement.

In the other hand, in fig.( $3 \mathrm{~d}-\mathrm{f})$ we can see that the $\mathrm{H}$ distributions have a maximum value at $\mathrm{t}=50 \mathrm{nmdepending}$ on the two modes of reflection at $\mathrm{t}=50 \mathrm{~nm}$ in fig. $(5 \mathrm{e})$.

Furthermore, these field enhancements are mainly located within the holes. This is highly desirable for biosensing devices as it rises the overlap of the analyses with the electromagnetic field $(\mathrm{E}$ and $\mathrm{H})$ inside the hole by effective the positions of the surface plasmons.
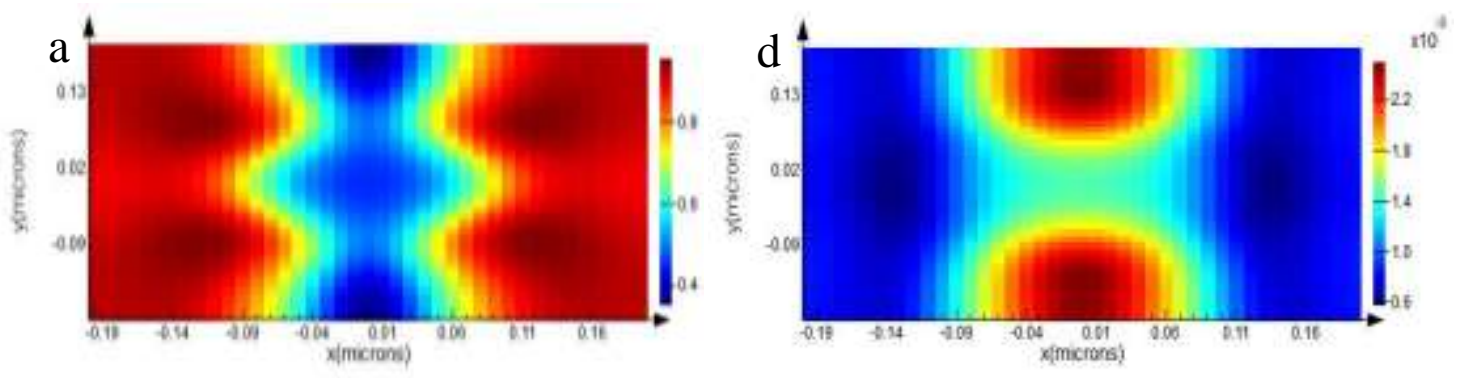

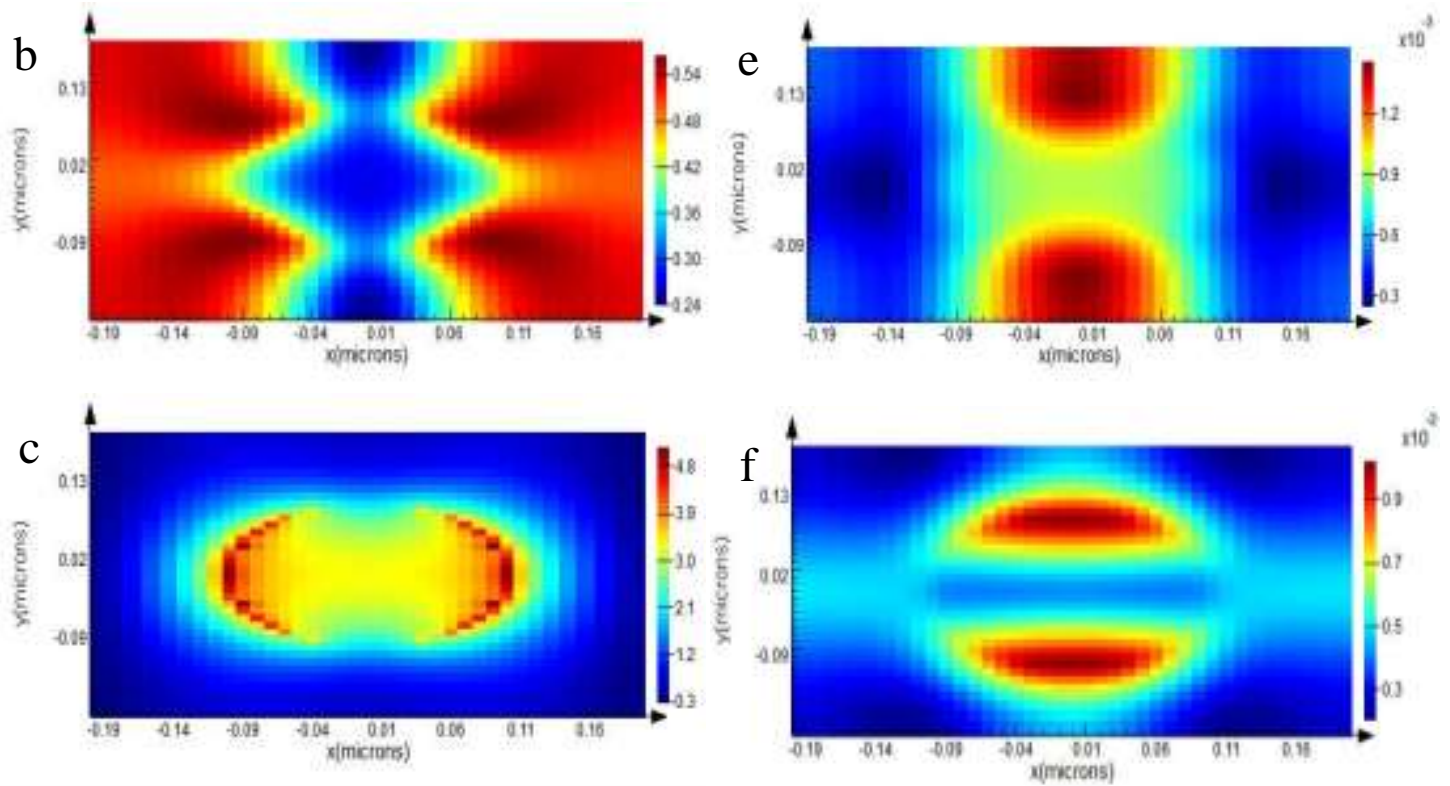

Fig 4. "Field Distributions for H and E Of 9-NHA with Varying Thickness of Au: $(20,50,100)$ Nm"

From fig.(4) the E distributions for 9-NHA with period $\mathrm{P}_{1}=300 \mathrm{~nm}$ are shown in fig.(4a-c),we can see that $\mathrm{E}$ at $\mathrm{t}=100 \mathrm{~nm}$ is larger than the other thickness $(20,50) \mathrm{nm}$ corresponding to the two modes of transmission in fig( $6 \mathrm{e})$ to determined the near field enhancement. In the other hand, in fig.(4d-f) we can see that the $\mathrm{H}$ distributions have a maximum value at $\mathrm{t}=100 \mathrm{~nm}$ depending on the two modes of reflection at $\mathrm{t}=100 \mathrm{~nm}$ in fig.( $6 \mathrm{~d}$ ).
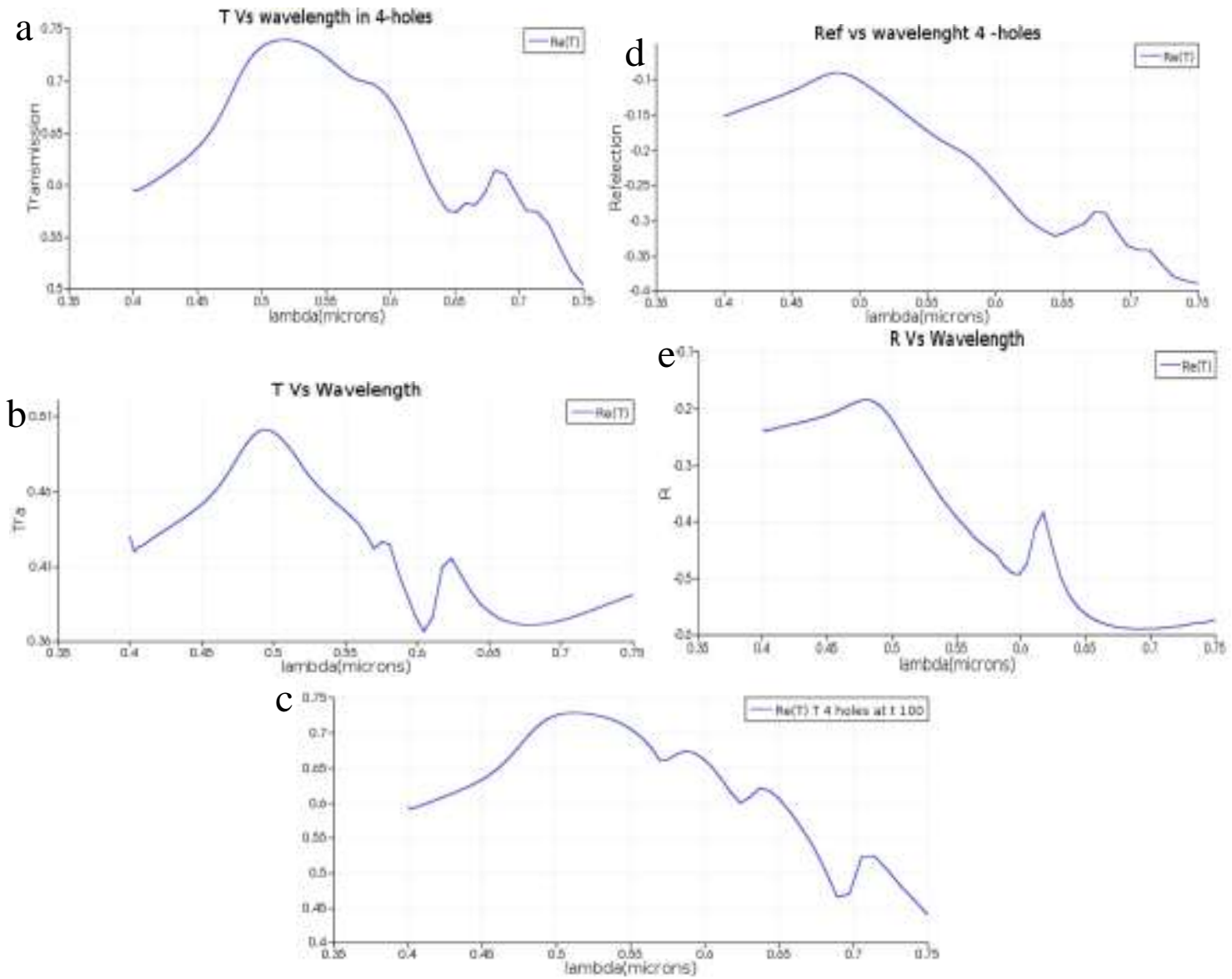

Fig 5. A, B And C “ Transmission; D and E Reflection Resonance for 4-NHA At $T(A u)=(20,50,100)$ Nm” 

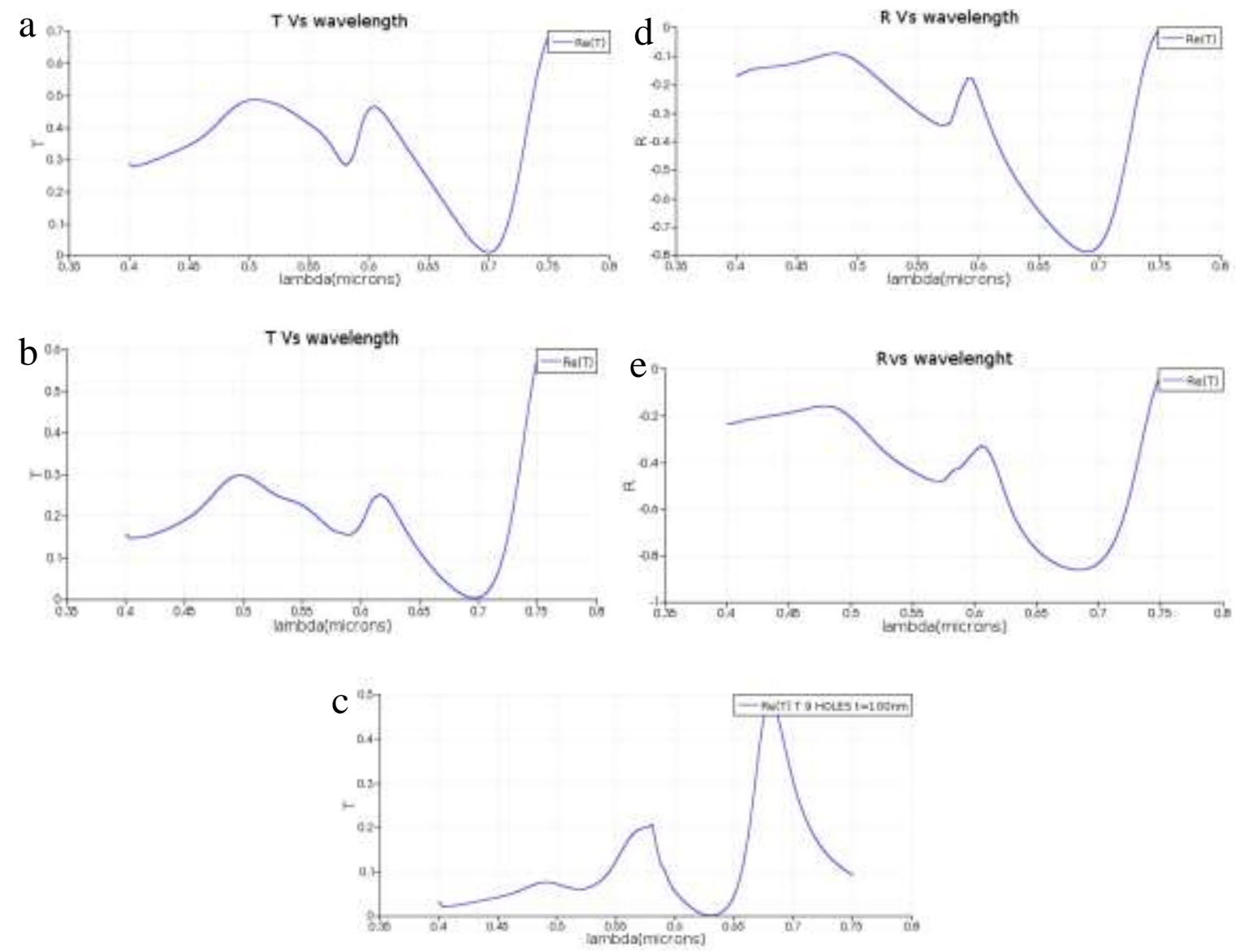

Fig 6. A, B And C “ Transmission; D and E Reflection Resonance for 9-NHA at T(Au) $=(20,50,100) \mathrm{Nm}$ ”

We can see that there is a peak value of the wavelength at $519 \mathrm{~nm}$ approximately to $73 \%$ strong light transmission with 4-NHA in fig.(5a), in the other hand wavelength of $519 \mathrm{~nm}$ transmission is $45 \%$ with 9NHA in fig.(6a). Strong light transmission is hopeful for using in biological devices with $\mathrm{Au} / \mathrm{SiO}_{2}$ interface .

\section{Conclusions}

In this article, we studied the important parameters that effect on the performance optical properties in NHA and surface plasmons like, the noble metals with dielectric layers; different geometrical of NHA with varying thickness and methods of excitation SPs.

From the results are observed the effect of holes depth, with consideration the number of holes and $\mathrm{P}$ have an influence on SPs interface, transmission is increased at $(\mathrm{t}=20 \mathrm{~nm})$ for two arrays but at 4-holes is greater than 9-holes array, that leads to which one is suitable for biosensors. With each thickness It is challenging to fabricate the nanoholes NHAs structure because the metal film is too thick for a clean liftoff (typically $\geq 100 \mathrm{~nm}$ for $\mathrm{Au}$ and $\geq 300 \mathrm{~nm}$ for $\mathrm{Ag}$ to make the film "optically thick").

\section{References}

[1] J.N. Anker, W.P. Hall, O. Lyandres, N.C. Shah, J. Zhao, and R.P. Van Duyne, "Biosensing with plasmonic nano sensors. Nature materials", 7(6):442-453, 2008. https://doi.org/10.1038/nmat2162

[2] Maier, S.” Plasmonics", New York: Springer ,2007.ch. 2,pp45. 
[3] Alexander G. Bro 1, Reuven Gordon, Brian Leathem, Karen L. Kavanagh, "Surface Plasmon Sensor Based on the Enhanced Light Transmission through Arrays of Nanoholes in Gold Films", Langmuir, 20, 4813-4815,. 2004. https://doi.org/10.1021/la0493621

[4] J.M. McMahon, J. Henzie, T.W. Odom, G.C. Schatz, S.K. Gr y, et al., « Tailoring the sensing capabilities of nanoholes arrays in gold films with Rayleigh anomaly-surface Plasmon Polariton”. Opt. Express, 15(26):18,. 2007. https://doi.org/10.1364/OE.15.018119

[5] R.B.M. Schasfoort and Anna J.," Handbook of Surface Plasmon Resonance”, 2008, ch1, PP38.

[6] [6] Antoine Lesueur, Nathan C. Lindquist, Kwan Seop Lim, and Sang-Hyun Oh., "Plasmonic nanoholes arrays for real-time multiplex biosensing",pp703504-10,2008.

[7] Andreas B. Dahlin.," Sensing applications based on plasmonic nonporous": The hole story. Analyst, 140:47484759,2015,. https://doi.org/10.1039/C4AN02258K

[8] Ebbesen TW, Lezec HJ, Ghaemi HF, Thio T, Wolff PA," Extraordinary optical transmission through sub-wavelength hole arrays.” Nature 391:667-669, 1998. https://doi.org/10.1038/35570

[9] L. Wu, P. Bai, X. Zhou,2 and E. P. Li," Reflection and Transmission Modes in Nanoholes-Array-Based Plasmonic Sensors", IEEE Photonics Journal, V 4, No 1,p26-33 February 2012. https://doi.org/10.1109/JPHOT.2011.2177652

[10] L. Martin-Moreno, FJ Garcia-Vidal, HJ Lezec, KM Pellerin, T. Thio, JB Pendry, and TW Ebbesen. ,’Theory of extraordinary optical transmission through subwavelength hole arrays". Physical review letters,86(6):1114-1117,2001. https://doi.org/10.1103/PhysRevLett.86.1114

[11] K. J. Klein Koerkamp, S. Enoch, F. B. Segerink, N. F. van Hulst, and L. Kuipers,"Strong influence of hole shape on extraordinary transmission through periodic arrays of subwavelength holes". Phys. Rev. Lett., 92:183901, May 2004. https://doi.org/10.1103/PhysRevLett.92.183901

[12] van der Molen KL, Koerkamp KJK, Enoch S, Segerink FB, van Hulst NF, Kuipers L, ," Role of shape and localized resonances in extraordinary transmission through periodic arrays of subwavelength holes: experiment and theory". Phys Rev B 72:045421,2005. https://doi.org/10.1103/PhysRevB.72.045421

[13] Rodrigo SG, García Vidal FJ,Martín-Moreno L, “Influence of material properties on extraordinary optical transmission through hole arrays". Phys Rev B 77:075401, 2008. https://doi.org/10.1103/PhysRevB.77.075401

[14] Cetin AE, Etezadi D, Galarreta BC, Busson MP, Eksioglu Y, Altug H, "Plasmonic nanoholes arrays on a robust hybrid substrate for highly sensitive label-free biosensing", ACS Photon 2:1167-1174,2015. https://doi.org/10.1021/acsphotonics.5b00242

[15] Je Hong Kim and Patrick J. Moyer, "Thickness effects on the optical transmission characteristics of small hole arrays on thin gold films", , Vol. 14, No. 15 / OPTICS EXPRESS 6595, 2006.

[16] Dhawan A, Vo-Dinh T." Theoretical simulation and focused ion beam fabrication of gold nanostructures for surfaceenhanced Raman scattering (SERS)". Nano bio technol. 3:164-71, 2007.

[17] Kunli Xiong and Andreas B. , "biosensing using plasmonic nano hole arrays with small, homogenous and tunable aperture diameters", Royal Society of Chemistry, 2017.

[18] Avijit Barik, Lauren M. Otto, Daehan Yoo,Jincy Jose, " Dielectrophoresis-Enhanced Plasmonic Sensing with Gold NanoholeArrays",dx.doi.org/10.1021/nl500149h | Nano Lett. 2014.

[19] A. B. Dahlin, P. Jonsson, M. P. Jonsson, E. Schmid , F. Hook, ACS Nano , 2 , 2174 , 2008. https://doi.org/10.1021/nn800254h

[20] Palik E.D ,"Handbook of optical constants of Solids “(academics press;1985).

[21] L. Wu, P. Bai, and E. P. Li, "Designing surface Plasmon resonance of subwavelength hole arrays by studying absorption", J. Opt. Soc. Amer. B, Opt. Phys., Dec. 2011. 